\title{
Disposable Chromatography for a High-Throughput Nano-ESI/MS and Nano-ESI/MS-MS Platform
}

\author{
Jason G. Williams and Kenneth B. Tomer \\ Laboratory of Structural Biology, National Institute of Environmental Health Sciences, National Institutes \\ of Health, Department of Health and Human Services, Research Triangle Park, North Carolina, USA
}

\begin{abstract}
High-throughput proteomics has typically relied on protein identification based on MALDI-MS peptide maps of proteolytic digests of 2D-gel-separated proteins. This technique, however, requires significant sequence coverage in order to achieve a high level of confidence in the identification. Tandem MS data have the advantage of requiring fewer peptides (2) for high confidence identification, assuming adequate MS/MS sequence coverage. MALDIMS/MS techniques are becoming available, but can still be problematic because of the difficulty of inducing fragment ions of a singly charged parent ion. Electrospray ionization, however, has the advantage of generating multiply charged species that are more readily fragmented during MS/MS analysis. Two electrospray/tandem mass spectrometry-based approaches, nanovial-ESI-MS/MS and LC-MS/MS, are used for high throughput proteomics, but much less often than MALDI-MS and peptide mass fingerprinting. Nanovial introduction entails extensive manual manipulation and often shows significant chemical background from the in-gel digest. LC-MS has the advantages that the chemical background can be removed prior to analysis and the analytes are concentrated during the separation, resulting in more abundant analyte signals. On the other hand, LC-MS can often be time intensive. Here, we report the incorporation of on-line sample clean-up and analyte concentration with a high-throughput, chip-based, robotic nano-ESI-MS platform for proteomics studies. (J Am Soc Mass Spectrom 2004, 15, 1333-1340) (c 2004 American Society for Mass Spectrometry
\end{abstract}

$\mathrm{M}$ ass spectrometry is the key element of highthroughput proteomics [1-5]. Electrospray ionization mass spectrometry (ESI-MS) [6] is well suited for protein identification from gel band digests because of the relative ease and availability of modern ESI-MS/MS techniques. Detection of far fewer peptides is necessary for unambiguous identification when tandem MS methods are incorporated than when only MS techniques are employed [7]. ESI-MS/MS has an advantage over matrix assisted laser desorption ionization (MALDI)-MS/MS [8] in that multiply charged species, which are more readily fragmented in tandem MS applications than singly charged species at equivalent collision energies, are formed during the electrospray process. Moreover, many labs are already equipped with mass spectrometers that are capable of ESI-MS/MS whereas MALDI instruments with tandem mass analyzers have become commercially available only in the last few years. Drawbacks of using ESI-MS or ESI-MS/MS as the final analytical step for protein identification, however, are the high chemical back-

Published online July 28, 2004

Address reprint requests to Dr. K. B. Tomer, Laboratory of Structural Biology, National Institute of Environmental Health Sciences, 111 TW Alexander Drive, P.O. Box 12233, MD-F0-03, Research Triangle Park, NC 27709, USA. E-mail: tomer@niehs.nih.gov ground and low sample abundance of proteolytic digests of gel bands. These problems can be overcome with on-line liquid chromatography (LC) or sample clean-up and concentration prior to ESI-MS, but neither of these techniques is conducive to high-throughput applications.

In an effort to achieve high-throughput clean-up for ESI-MS and ESI-MS/MS analysis of peptides from gel band tryptic digests, we have adapted several approaches for concentrating and desalting these tryptic peptides for implementation with a robotic nanospray system, the Advion Biosciences Nanomate 100 [9-11]. The first of these techniques involves modifying the conductive graphite pipette tips of the Nanomate system by inserting a fiberglass frit into the pipette tip and then adding a bed volume of approximate $10 \mu \mathrm{L}$ of Poros $50 \mathrm{R} 2$ resin. Poros resins have been used off-line with much success for both concentrating and desalting gel digests $[12,13]$. The second approach is to modify the commercially available ZipTips from Millipore with a conductive electrode so they can be used directly on the Nanomate high-throughput platform. Much like the Poros resins, ZipTips have shown their utility for desalting and concentrating peptide, as well as protein samples $[10,14,15]$. The final approach is to modify the Nanomate itself such that a solvent gradient can be 
delivered from a liquid chromatography system through the Nanomate and across a disposable, either self-packed or commercial, chromatographic pipette tip.

\section{Experimental}

\section{Materials and Reagents}

Renin substrate tetradecapeptide, bovine ubiquitin, and bovine serum albumin are all products of Sigma-Aldrich (St. Louis, MO). All other chemicals and reagents were also purchased from Sigma-Aldrich unless otherwise noted. Poros resin is a product of Applied Biosystems (Framingham, MA). ZipTips were purchased from Millipore (Billerica, MA). All metals are products of Scientific Instrument Services, Inc (Ringoes, NJ).

\section{Chip and Mass Spectrometry Systems}

A high-throughput, chip-based nanospray system, the Nanomate 100 from Advion Biosciences (Ithaca, NY), was used for this work. With this system, each sample is processed using a separate pipette tip and chip nozzle, eliminating carryover between samples. A sample-filled tip aligns with a chip nozzle, creating a seal. Nano-ESI initiates when head pressure and voltage are applied through the graphite sample tip. The mass spectrometer used was a Micromass QTOF micro hybrid mass spectrometer (Micromass/Waters, Milford, MA).

The Micromass QTOF micro hybrid mass spectrometer instrumental conditions were: Positive ion mode; scan range of 300 to $2000 \mathrm{~m} / \mathrm{z}$; scan rate of 1 scan per s for MS and 1 scan per $2.5 \mathrm{~s}$ for MS/MS; sample cone voltage of $40 \mathrm{~V}$; source temperature of $80{ }^{\circ} \mathrm{C}$; cone gas at $50 \mathrm{~L} / \mathrm{h}$; desolvation gas at $100 \mathrm{~L} / \mathrm{h}$ (necessary for Nanomate 100 operation). Collision energy was $10 \mathrm{~V}$ for MS analyses and $27 \mathrm{~V}$ for MS/MS analyses. The external gauge setting for the gas cell pressure was maintained at 13 psi with nitrogen gas. The Nanomate 100 was operated in the positive ion mode with a gas pressure ranging from 0.3 to $1.5 \mathrm{psi}$ and a probe voltage ranging from 1.5 to $2.0 \mathrm{kV}$.

Data were analyzed with the MassLynx suite of software (Micromass/Waters, Milford, MA). All spectra were a sum of 20 individual scans, unless otherwise noted, and expressed as ion abundance relative to the ion abundance of the base peak in each individual spectrum.

\section{Chromatographic Pipette Tips}

Chromatographic pipette tips were made from either the $10 \mu \mathrm{L}$ graphite pipette tips offered by Advion Biosciences or standard $10 \mu \mathrm{L}$ pipette tips filled with Poros resin. First a small home-built fiberglass frit was inserted into a $10 \mu \mathrm{L}$ pipette tip. Next, $20 \mu \mathrm{L}$ of 50:25:25 Poros $50 \mathrm{R} 2$ resin: $0.2 \%$ formic acid:acetonitrile slurry was packed into the tip by passing 50 bed volumes of

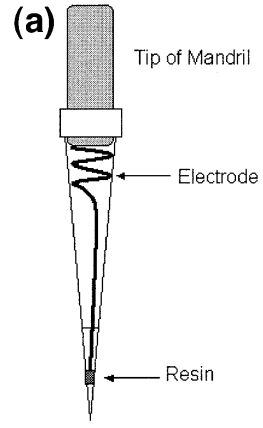

(b)

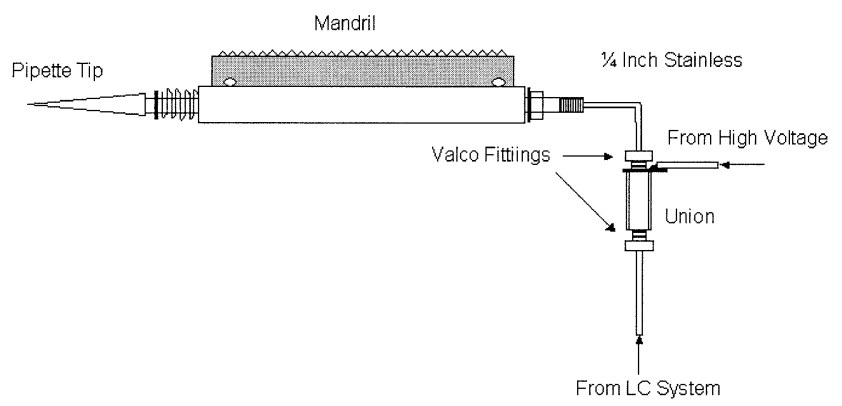

Figure 1. $\mathrm{C}_{18}$ Millipore ZipTips fitted with electrode (a) and the mandril from the Nanomate 100 system with LC modifications (b). The mandril was removed from the instrument and the Teflon tubing plumbing was removed. Stainless steel (1/16 inch) HPLC tubing was threaded through the center bore of the mandril. HPLC fittings and the high voltage leads are also attached to this stainless steel tubing.

50:50 $0.2 \%$ formic acid: acetonitrile through the tip using an Eppendorf $2.5 \mathrm{~mL}$ multipette tip.

Millipore ZipTips $\left(\mathrm{C}_{4}, \mathrm{C}_{18}\right.$, and SCX) were fitted with a spring-shaped metal wire with a lead extending to the resin bed (Figure 1a). The wires were made of copper, gold, platinum, or tungsten. These electrodes were fashioned by wrapping 5-10 turns of the wire around the outside of a $10 \mu \mathrm{L}$ pipette tip to form a coil, that is then stretched into a slightly extended spring that is then inserted into the pipette tip so that the extending lead makes physical contact with the resin bed inside the ZipTip. In addition, the spring extends up toward the probe seating area of the pipette tip so that it contacts the high voltage supplying mandril of the Nanomate system.

\section{Sample Preparation}

Renin substrate tetradecapeptide (RSTP) was used at concentrations varying from 500 picomolar to 1 micromolar in either $50: 500.2 \%$ formic acid:acetonitrile, 20 $\mathrm{mM}$ Tris and $500 \mathrm{mM} \mathrm{NaCl}$, or $1 \%$ glycerol. Ubiquitin was used at 1 micromolar in either 50:50 0.2\% formic acid: acetonitrile or $250 \mathrm{mM} \mathrm{NaCl}$. Samples were loaded and washed three times off-line with $10 \mu \mathrm{L}$ of $0.2 \%$ formic acid in water and then eluted on-line with 40:60 $0.2 \%$ formic acid:acetonitrile. $100 \mu \mathrm{L}$ of $1 \mathrm{mg} / \mathrm{mL}$ bo- 


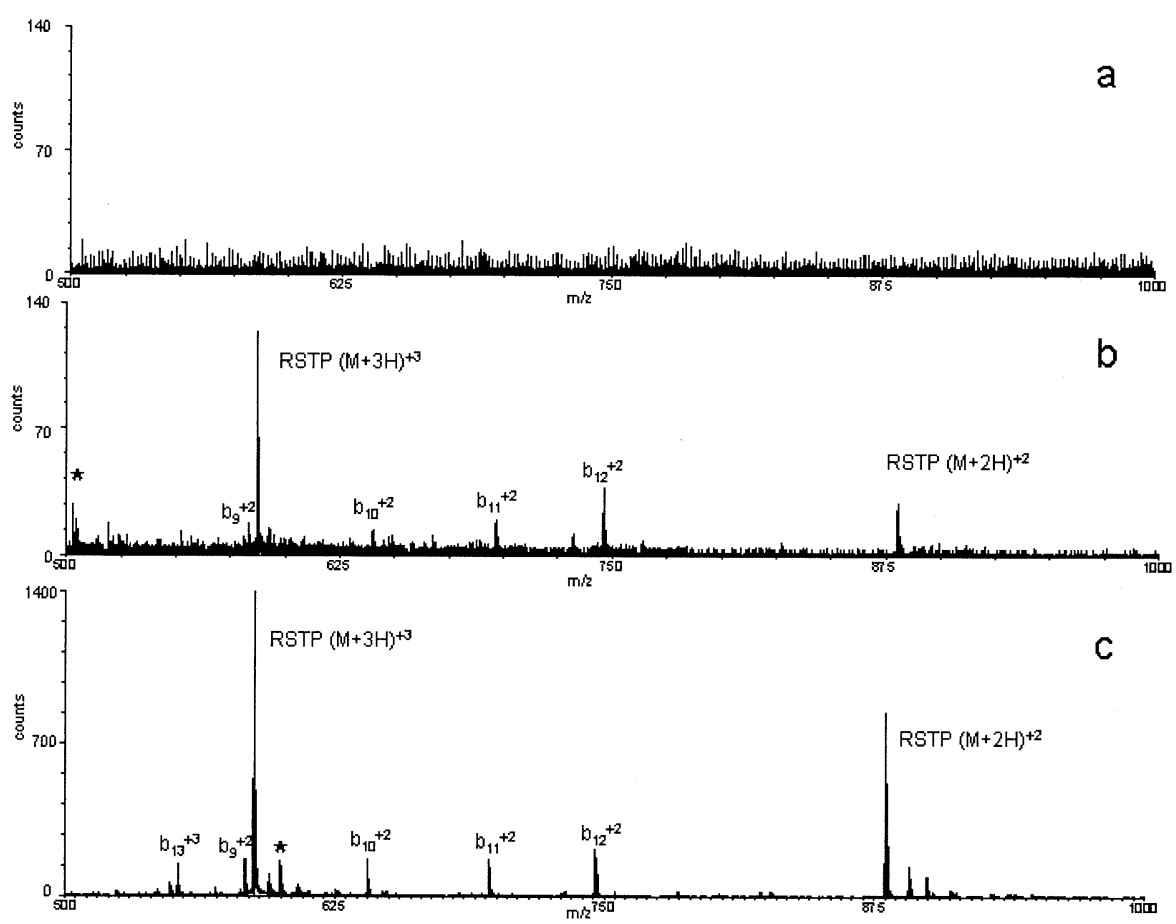

Figure 2. ESI mass spectrum of $5 \mu \mathrm{L}$ of $1 \mu \mathrm{M}$ RSTP in $20 \mathrm{mM}$ Tris (pH 7.5) and $500 \mathrm{mM} \mathrm{NaCl}$ analyzed using either the standard Nanomate graphite tips (a) or desalted using the Poros filled graphite pipette tips (b). A representative ESI mass spectrum of $50 \mathrm{nM}$ RSTP in 50:50 0.2\% formic acid:acetonitrile sprayed from the Nanomate 100 (c). Some fragments ions of RSTP are observed due to source fragmentation. This phenomenon is often observed in our laboratory with both the Micromass/Waters nanospray ESI-source as well as the Advion Biosciences Nanomate 100. An asterisk denotes unidentified ion.

vine serum albumin (BSA) was incubated for $12 \mathrm{~h}$ at $37^{\circ} \mathrm{C}$ with $1 \mu \mathrm{g}$ methylated trypsin (Promega, Madison, $\mathrm{WI})$.

\section{Nanomate Modifications for LC Compatability}

In order to accommodate the Agilent Cap1100 LC system, the Nanomate was first modified by overriding all safety contact closures such that the instrument could be operated without its primary cover. Because removing the cover of the instrument exposes the user to possible electric shock hazard, careful attention was paid to insulate the high voltage connections and to shield the sample stage area. The Nanomate mandril was removed from the instrument and the Teflon tubing for the nitrogen gas system was removed and replaced with stainless steel HPLC tubing $(1 / 16$ inch $\times$ $15 \mathrm{~cm})$. Valco fittings and a Supelco butt connector were used to connect the stainless steel plumbing to the HPLC pumps. To apply high voltage to the analyte, the spray needle high voltage lead was attached to the stainless steel at the butt connector union. (Figure 1b).

\section{Chromatography}

The Agilent (Palo Alto, CA) 1100 capillary liquid chromatography system was used at a flow-rate of $1 \mu \mathrm{L} /$ min with a gradient (Buffer A: $0.1 \%$ formic acid in water; Buffer B: $0.1 \%$ formic acid in acetonitrile) from $2 \%$ B to $70 \%$ B over 10 min and then from $70 \%$ B to $95 \%$ B over 2 min and held at $95 \%$ B for $15 \mathrm{~min}$.

\section{Results and Discussion}

\section{Poros Filled Graphite Tips}

As proof of principle, $5 \mu \mathrm{L}$ of $1 \mu \mathrm{M}$ renin substrate tetradecapeptide (RSTP) in $20 \mathrm{mM}$ Tris and $500 \mathrm{mM}$ $\mathrm{NaCl}$ were analyzed by ESI-MS using either the standard Nanomate graphite tips or the modified desalting pipette tips. For the ESI-MS with the standard tips, no ions that corresponded in mass to RSTP where observed (Figure 2a), but when the desalting tips where utilized (Figure 2b), abundant ions of $\mathrm{m} / \mathrm{z} 879.8$ and 586.9 , corresponding to doubly and triply charged RSTP molecular ions, were observed. Figure $2 \mathrm{c}$ is a representative ESI spectrum of RSTP collected via flow injection from a $0.5 \mu \mathrm{M}$ RSTP sample (500 femtomoles / $\mu \mathrm{L}$ at approximately $100 \mathrm{~nL} / \mathrm{min}$ ) in 50:50 $0.2 \%$ formic acid in water:acetonitrile solution. Note that, although the spectrum of the desalted RSTP sample (Figure $2 \mathrm{~b})$ contains peaks corresponding to the $(\mathrm{M}+$ $2 \mathrm{H})^{+2}$ and $(\mathrm{M}+3 \mathrm{H})^{+3}$ charge states at moderate abundance (140 counts), the RSTP sample in 50:50 0.2\% formic acid:acetonitrile (Figure 2c) has a significantly higher absolute ion abundance at the same concentra- 

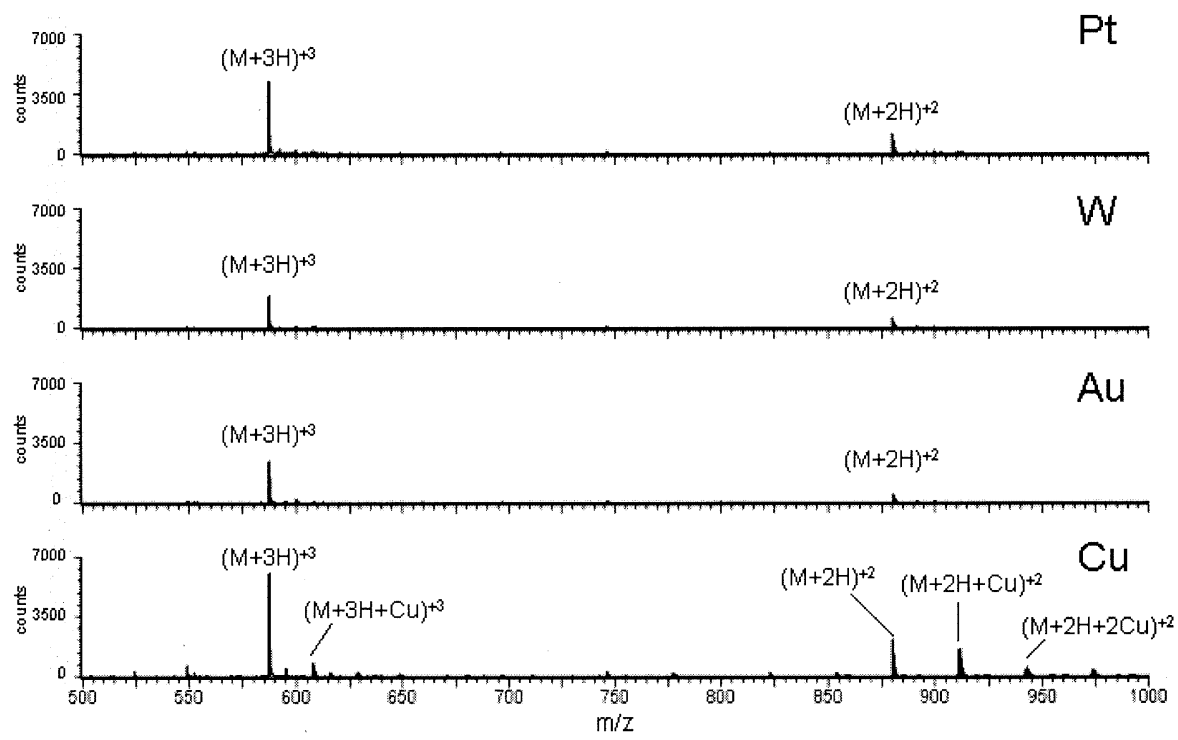

Figure 3. ESI Mass spectra of RSTP sprayed from $\mathrm{C}_{18}$ ZipTips fitted with electrodes composed of different metals. $5 \mu \mathrm{L}$ of $1 \mu \mathrm{M}$ RSTP in $20 \mathrm{mM}$ Tris (pH 7.5) and $500 \mathrm{mM} \mathrm{NaCl}$ were captured on $\mathrm{C}_{18}$ ZipTips and then eluted directly into the mass spectrometer using the Nanomate 100.

tion of RSTP. It is difficult to determine if this decrease in ion abundance is due to inefficient capture/release of the peptide during the desalting process, if the desalting process was incomplete resulting in ion suppression of the RSTP ions, or if the ionization process was less efficient when performing ESI with the homemade tips. Although ESI is usually more sensitive at lower flow rates, hence the advent of nano-ESI techniques, the lower ion abundance observed in Figure $2 \mathrm{~b}$ may also be attributed to an additional complication in that the resin-filled pipette tips have a much lower flow rate relative to blank tips at a given nitrogen gas pressure. In fact, an increase in sensitivity can be observed when the nitrogen gas head pressure is increased on the Nanomate system (data not shown).

\section{$C_{18}$ ZipTips}

Although the Poros packed graphite tips perform well with the Nanomate system, it is time consuming and difficult to fabricate these tips in a reproducible manner. Moreover, the observed ion abundances for these tips are lower than what one would expect for a given amount of analyte, for possible reasons previously mentioned. We, therefore, tested commercially available Millipore ZipTips with the Nanomate system. To provide high voltage to the analyte, metal electrodes composed of copper ( 0.015 inch diameter), gold $(0.025$ inch diameter), platinum (0.008 inch diameter), or tungsten (0.01 inch diameter) were fabricated and placed into the tips (Figure 1a). $5 \mu \mathrm{L}$ of 1 nanomolar RSTP in 20 $\mathrm{mM}$ Tris, $500 \mathrm{mM} \mathrm{NaCl}$ were adsorbed onto the $\mathrm{C}_{18}$ ZipTips, washed, and the solute eluted directly into the mass spectrometer by the addition of $5 \mu \mathrm{L}$ of $40: 600.2 \%$ formic acid:acetonitrile. Figure 3 shows the corresponding mass spectra of the RSTP solution following desalt- ing and elution from the ZipTips fitted with the four different metal electrodes. In all cases, the $(\mathrm{M}+2 \mathrm{H})^{+2}$ and $(\mathrm{M}+3 \mathrm{H})^{+3}$ ions of RSTP were observed. In addition, ions corresponding to copper adducts of RSTP (addition of $\mathrm{m} / \mathrm{z} 31.5$ for the $(\mathrm{M}+2 \mathrm{H})^{+2}$ charge state and $m / z 21$ for the $(\mathrm{M}+3 \mathrm{H})^{+3}$ charge state) were observed when the copper electrodes were used. Because MS data collected from ZipTips with platinum electrodes consistently, although modestly, had higher absolute counts, platinum electrodes where used for all other experiments.

The platinum electrode-modified $\mathrm{C}_{18}$ ZipTips are also suitable for ESI-MS/MS applications. Figure 4 shows an ESI-MS/MS spectrum, transformed to singly charged ions (MaxEnt Masslynx, Micromass/Waters, Milford, MA), of the $(\mathrm{M}+2 \mathrm{H})^{+2}$ ion of $m / z 586.9$ from a solution containing 1 nanomolar RSTP in $20 \mathrm{mM}$ Tris, $500 \mathrm{mM} \mathrm{NaCl}$ following elution using the on-line desalting technique. Specifically, $10 \mu \mathrm{L}$ of the above solution, corresponding to 10 femtomoles RSTP, were captured on a $\mathrm{C}_{18}$ ZipTip, washed three times with $10 \mu \mathrm{L}$ of $0.1 \%$ formic acid in water, and then eluted directly into the mass spectrometer with $5 \mu \mathrm{L}$ of $40: 600.2 \%$ formic acid: acetonitrile. Many fragment ions can be observed. Specifically, an a-series [16], $a_{6}$ through $a_{12}$, and a b-series, $b_{4}$ through $b_{12}$, are seen. This is very consistent with the fragmentation pattern normally observed for RSTP on our QTOF micro mass spectrometer. Moreover, the data collected from the desalting pipette tips was comparable in ion abundance to data collected via normal nano-ESI using an equal amount of RSTP.

To demonstrate the ability of these tips to concentrate a dilute sample, a 250 picomolar RSTP sample (0.25 femtomoles $/ \mu \mathrm{L}$ in 50:50 $0.2 \%$ formic acid:acetonitrile) was analyzed with the QTOF micro via the Nanomate using either standard graphite tips or platinum 

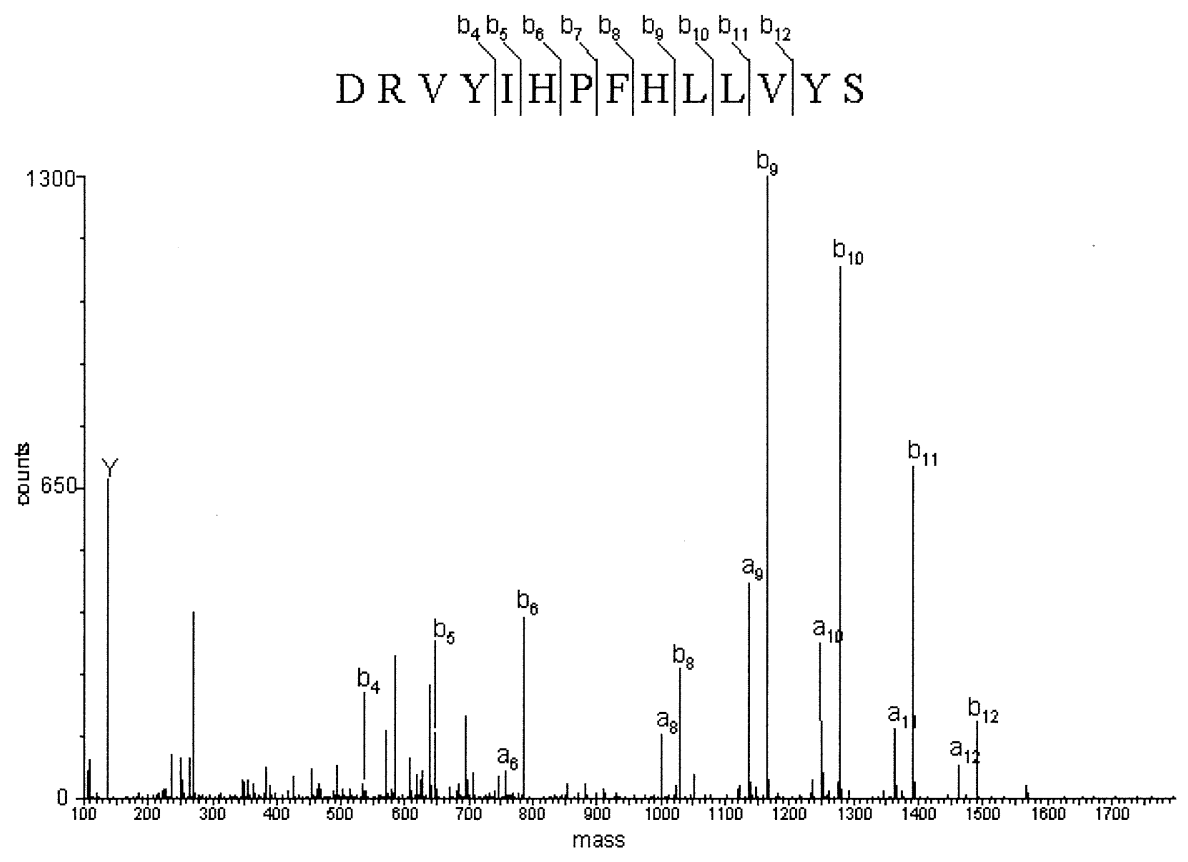

Figure 4. Deconvoluted mass spectrum of a tandem MS spectrum of RSTP from a $\mathrm{C}_{18}$ ZipTip. Tandem MS was performed on the triply charged $m / z 586.9$ ion of RSTP with a collision energy of $27 \mathrm{~V}$.

electrode-modified $\mathrm{C}_{18}$ ZipTips (Figure $5 \mathrm{a}$ and b). For the $(\mathrm{M}+3 \mathrm{H})^{+3}$ ion, 17 counts were observed when summing two individual mass spectra from this analysis. Ten microliters of 250 picomolar (2.5 femtomoles) RSTP in water was captured by loading the sample onto a $\mathrm{C}_{18}$ ZipTip. The sample was then eluted directly into the mass spectrometer by the addition of $5 \mu \mathrm{L}$ of 40:60 $0.2 \%$ formic acid:acetonitrile. For the $(\mathrm{M}+3 \mathrm{H})^{+3}$ ion, 57 counts were observed when summing two individual mass spectra. These data demonstrate that absolute counts were increased approximately 3.5 -fold with this method while the overall signal to noise ratio was increased from approximately 2.5 to approximately 14 , nearly a 6-fold increase. It should be noted that the RSTP ion abundance quickly diminished to near zero counts in approximately $20 \mathrm{~s}$ as the peptide was eluted from the tip matrix.

\section{SCX ZipTips}

To demonstrate the versatility of our approach, the strong cation exchange SCX ZipTip was also tested.
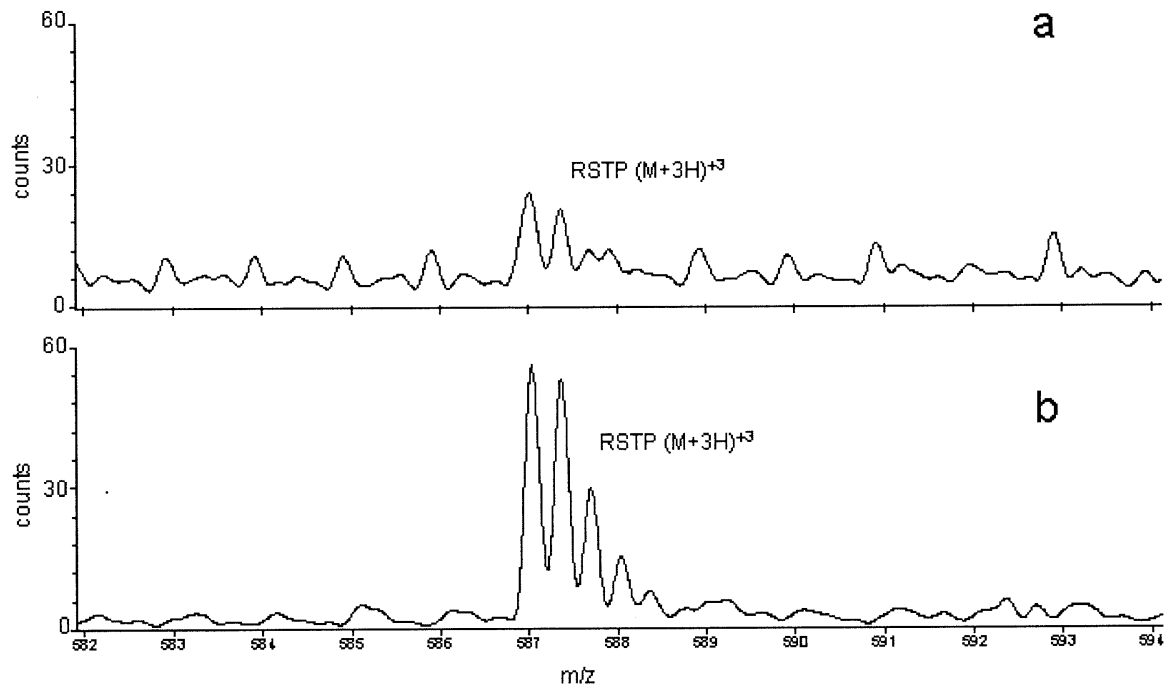

Figure 5. 250 picomolar RSTP in 50:50 0.2\% formic acid:acetonitrile was analyzed with ESI-MS via the Nanomate 100 with standard graphite tips (a) or captured onto and then eluted and sprayed from the Nanomate 100 by the addition of 40:60 0.2\% formic acid:acetonitrile (b). 


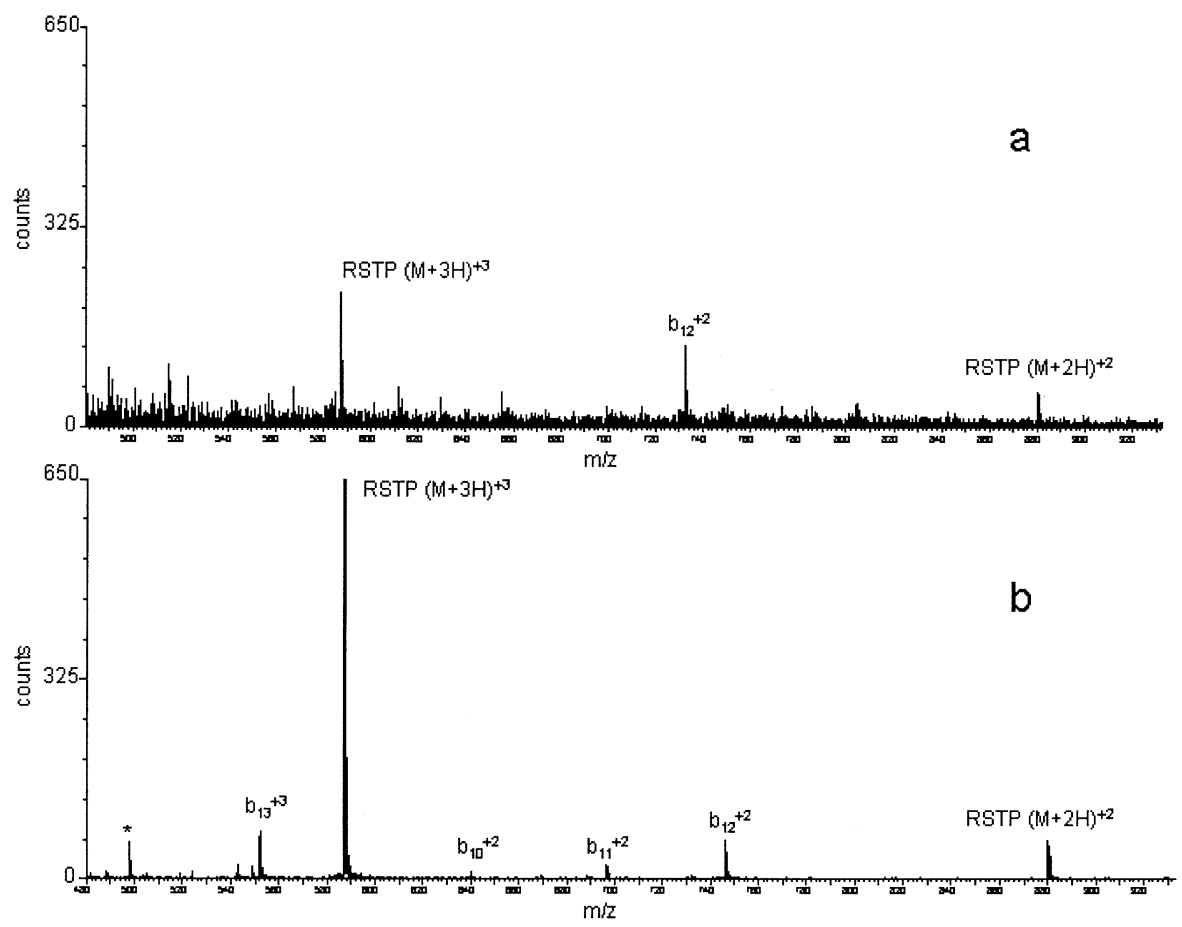

Figure 6. SCX ZipTips for removal of non-polar contaminants. ESI mass spectrum of RSTP in 49.5:49.5:1 0.2\% formic acid:acetonitrile:glycerol sprayed from the standard graphite tip (a). ESI mass spectrum of the same RSTP sample after clean-up with a Millipore SCX ZipTip fitted with a platinum electrode (b).

Figure 6a shows the ESI-MS analysis of 1 nanomolar RSTP in 49.5:49.5:1 0.2\% formic acid:acetonitrile:glycerol via the Nanomate without on-line desalting. Although ions that correspond in mass to RSTP are observed at $m / z 586.9$ and 879.9 despite the presence of glycerol, the ion abundance is relatively low and singly charged polymeric (repeating units of $44 \mathrm{Da}$ ) ions can also be observed. Figure $6 \mathrm{~b}$ shows the ESI mass spectrum of the same RSTP sample after clean-up with a Millipore SCX ZipTip (strong cation exchange) fitted with a platinum electrode. Even though this sample was eluted from the SCX tip with 40:60 0.2\% $\mathrm{NH}_{4} \mathrm{OH}$ :acetonitrile, the Nanomate and QTOF micro were both still operated in the positive ion mode for this analysis and the $(\mathrm{M}+2 \mathrm{H})^{+2}$ and $(\mathrm{M}+3 \mathrm{H})^{+3}$ charge states of RSTP are readily observed.

\section{$\mathrm{C}_{4}$ ZipTips}

In addition to the ESI-MS of peptides, the ability to perform ESI-MS analyses of undigested proteins in MS incompatible buffers is also of interest. Therefore, we also modified $\mathrm{C}_{4}$ ZipTips with platinum electrodes and tested their on-line desalting capabilities using the 8 $\mathrm{kDa}$ protein ubiquitin. Five $\mu \mathrm{L}$ of $1 \mu \mathrm{M}$ ubiquitin (5 picomoles) in $20 \mathrm{mM}$ Tris and $250 \mathrm{mM} \mathrm{NaCl}$ were analyzed by ESI-MS using either the standard Nanomate graphite tips or the electrode-modified Millipore $\mathrm{C}_{4}$ ZipTips. With the standard tips essentially no ions were observed (Figure 7a). When the Millipore $C_{4}$
ZipTips were utilized (Figure $7 \mathrm{~b}$ ), however, relatively abundant ions were observed for the ubiquitin $(\mathrm{M}+$ $7 \mathrm{H})^{+7}$ through the ubiquitin $(\mathrm{M}+12 \mathrm{H})^{+12}$ charge states. Figure $7 \mathrm{c}$ is a representative mass spectrum of ubiquitin acquired from the Nanomate using $5 \mu \mathrm{L}$ of 1 $\mu \mathrm{M}$ ubiquitin (5 picomoles) in 50:50 0.2\% formic acid: acetonitrile. A second charge envelope can be observed embedded within the ubiquitin charge envelope in both Figure $7 \mathrm{~b}$ and Figure 7c. This second charge envelope corresponds to the $(\mathrm{M}+6 \mathrm{H})^{+6}$ through the $(\mathrm{M}+9 \mathrm{H})^{+9}$ charge states of a truncated form of ubiquitin $\left(\mathrm{M}_{\mathrm{r}}=\right.$ $6532 \mathrm{Da}$ ) which corresponds to residues 19-76.

\section{Liquid Chromatography}

We have employed our direct spray tips for a crude gradient elution liquid chromatography as well. The Nanomate system was first retrofitted with stainless steel HPLC tubing as described in the Materials and Methods section. Standard $10 \mu \mathrm{L}$ disposable pipette tips were filled with approximate a $30 \mu \mathrm{L}$ bed volume of Poros resin. Then, $10 \mu \mathrm{L}$ of $1 \mu \mathrm{g} / \mu \mathrm{L}$ BSA tryptic digest were loaded onto the disposable chromatographic tips and off-line. After an on-line wash, a solvent gradient was then delivered to the pre-loaded pipette tip while performing ESI-MS from the Nanomate system. Figure 8 displays the total ion chromatogram and several single ion chromatograms (SIC) from a representative LC-ESI-MS analysis. Single ion chromatograms are of 

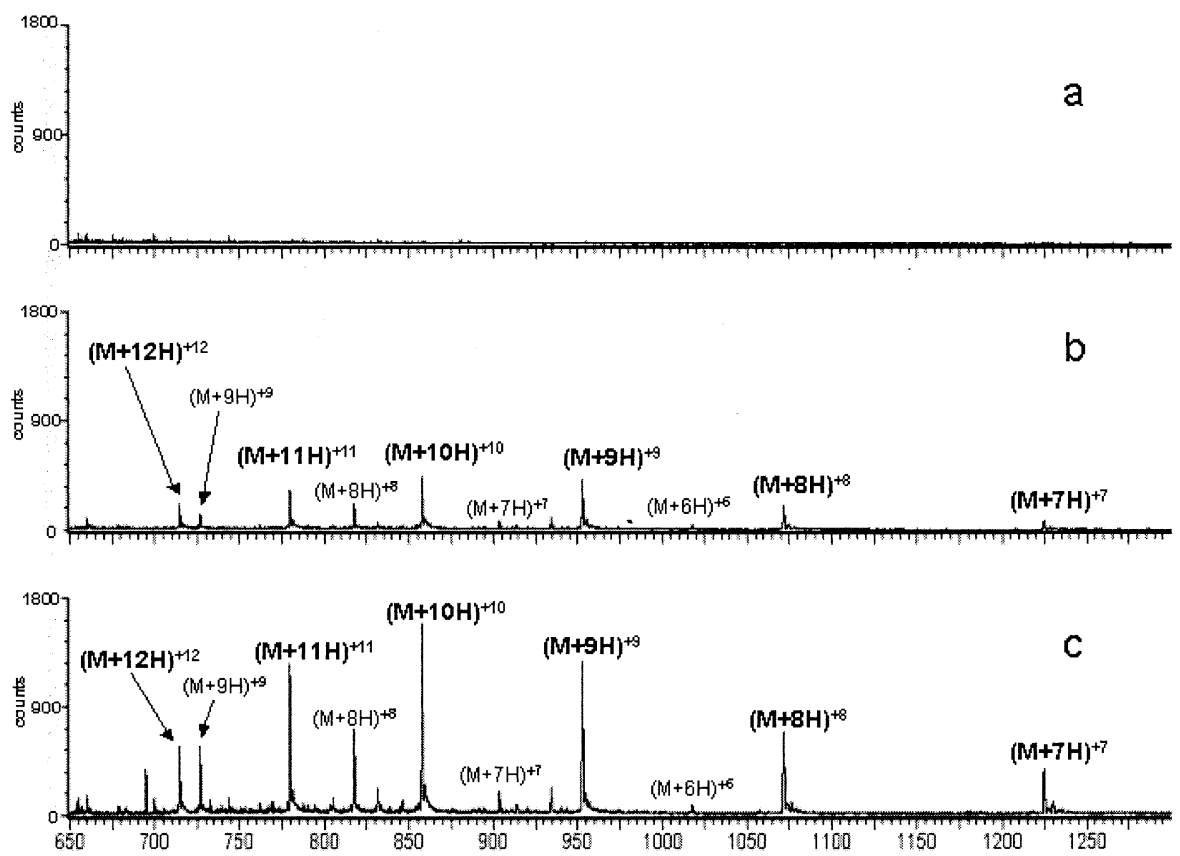

Figure 7. On-line desalting of ubiquitin with $\mathrm{C}_{4}$ ZipTips. ESI mass spectrum of $5 \mu \mathrm{L}$ of $1 \mu \mathrm{M}$ ubiquitin in $20 \mathrm{mM}$ Tris and $250 \mathrm{mM} \mathrm{NaCl}$ analyzed using either the standard Nanomate graphite tips (a) or the electrode-modified Millipore $\mathrm{C}_{4}$ ZipTips (b). Representative mass spectrum of $1 \mu \mathrm{M}$ ubiquitin in 50:50 0.2\% formic acid:acetonitrile from the Nanomate 100 (c). The bold labels correspond to full-length ubiquitin $\left(\mathrm{M}_{\mathrm{r}}=8565\right)$ while the normal typeface labels correspond to a truncated form of ubiquitin $\left(\mathrm{M}_{\mathrm{r}}=6532\right)$.

tryptic fragment 5-6 (one missed cleavage; residues 25-34; DTHKSEIAHR), fragment 21 (residues 157-160; FWGK), and fragment 63 (residues 452-455; SLGK). All three peptides had elution windows of roughly $15 \mathrm{~min}$. One potential advantage of the extended elution win- dows is increased time for MS/MS acquisitions. Although far from optimum chromatographic resolution was obtained from these first "proof of principle" analyses, this experiment demonstrates that this system can be modified for use with essentially "disposable" columns.
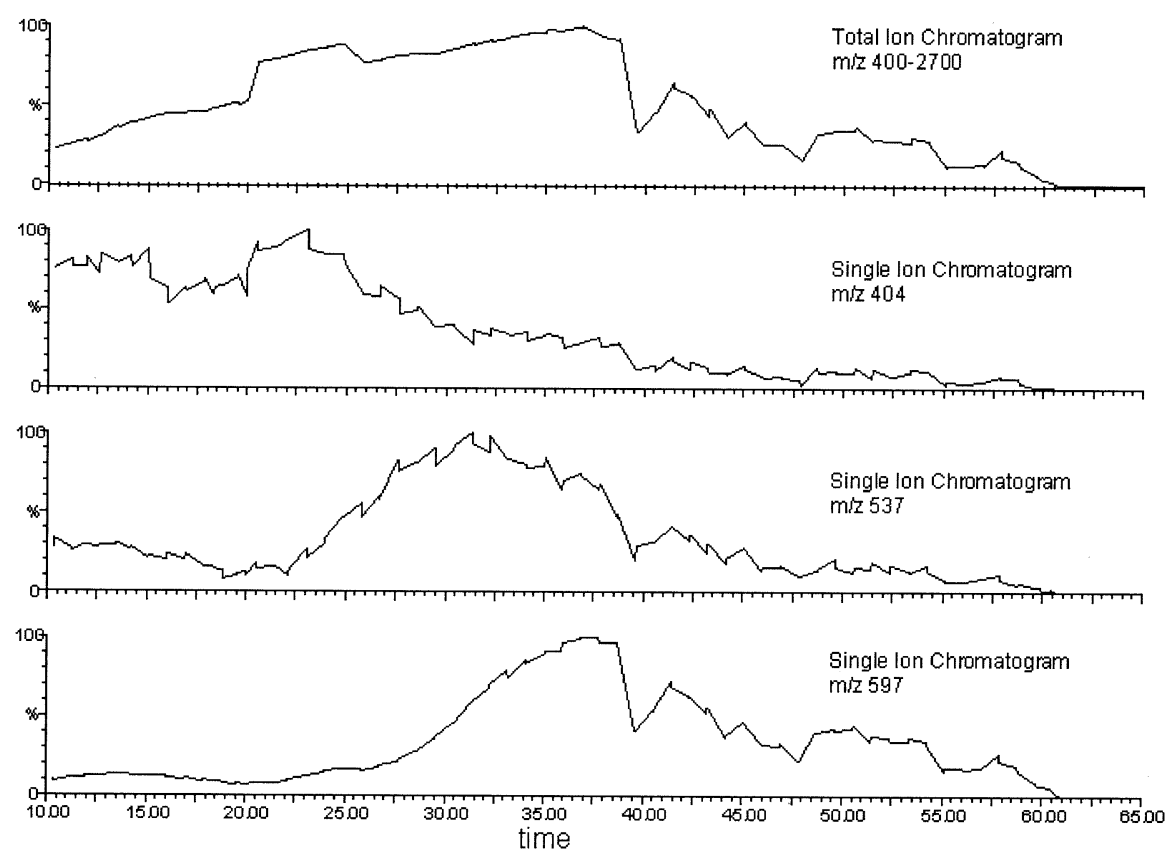

Figure 8. Total ion chromatogram and single ion chromatograms of a BSA tryptic digest using disposable liquid chromatography. 


\section{Conclusions}

The process of sample clean-up prior to conducting ESI-MS experiments is often both time and sample consuming. Here we report a method where nanoESI-MS and nano-ESI-MS/MS can be performed directly from chromatographic pipette tips on a highthroughput ESI-MS and MS/MS platform. The Nanomate 100 from Advion Biosciences is a platform for performing high-throughput ESI-MS and ESI-MS/ MS. It does, however, have the disadvantage in that most biological samples are highly contaminated by buffers, salts, detergents, etc. that are not amenable to ESI. The initial version of the Nanomate does not have the capability of high-throughput sample clean-up and, hence, performs best with samples containing little or no non-volatile salts. In an effort to enable the Nanomate 100 to be used in a high-throughput manner with salt or detergent contaminated samples, several types of chromatographic and/or de-salting tips were generated based on commercially available technologies. The Nanomate graphite tips supplied by Advion Biosciences can be packed with resins or Millipore ZipTips can be fitted with electrodes and both can then be used directly on the chip-based platform. Both types of these chromatographic tips work well with this system for sample clean-up of peptides and proteins. In this paper, the use of $\mathrm{C}_{18}$ ZipTips for concentration of a dilute sample, SCX ZipTips for the removal of glycerol from a standard sample, and $\mathrm{C}_{4}$ ZipTips for protein applications was demonstrated. In the future, incorporation of robotic methods for loading and washing the tips will be undertaken in efforts for high-throughput on-line sample clean-up and protein identification via ESI-MS and ESI-MS/MS. This system and approach should also be compatible with metal chelating tips from Millipore (MC ZipTips) for identification of phosphopeptides. The utility of the MC ZipTips as well as the performance of other chromatographic pipette tip technologies offered by other suppliers is currently being explored.

\section{References}

1. Aebersold, R.; Cravatt, B. F. Proteomics-Advances, Applications, and the Challenges that Remain. Trends Biotechnol. 2002, 20(12), S1-2.

2. Aebersold, R.; Mann, M. Mass Spectrometry-Based Proteomics. Nature 2003, 422(6928), 198-207.
3. Peng, J.; Gygi, S. P. Proteomics: The Move to Mixtures. J Mass Spectrom. 2001, 36(10), 1083-1091.

4. Patterson, S. D.; Aebersold, R. H. Proteomics: The First Decade and Beyond. Nat. Genet. 2003, 33(suppl), 311-323.

5. Yates, III. J. R., Mass Spectrometry. From Genomics to Proteomics. Trends Genet. 2000, 16(1), 5-8.

6. Fenn, J. B.; Mann, M.; Meng, C. K.; Wong, S. F.; Whitehouse, C. M. Electrospray Ionization for Mass Spectrometry of Large Biomolecules. Science 1989, 246(4926), 64-71.

7. Zeng, R.; Chen, Y. B.; Shao, X. X.; Shieh, C. H.; Miller, K.; Tran, H.; Xia, Q. C. Identification of Proteins Separated by OneDimensional Sodium Dodecyl Sulfate/Polyacrylamide Gel Electrophoresis with Matrix-Assisted Laser Desorption/Ionization Ion Trap Mass Spectrometry; Comparison with MatrixAssisted Laser Desorption/Ionization Time-of-Flight Mass Fingerprinting. Rapid Commun. Mass Spectrom. 2003, 17(17), 1995-2004.

8. Karas, M.; Hillenkamp, F. Laser Desorption Ionization of Proteins with Molecular Masses Exceeding 10,000 daltons. Anal. Chem. 1988, 60(20), 2299-2301.

9. Kapron, J. T.; Pace, E.; Van Pelt, C. K.; Henion, J. Quantitation of Midazolam in Human Plasma by Automated Chip-Based Infusion Nanoelectrospray Tandem Mass Spectrometry. Rapid Commun. Mass Spectrom. 2003, 17(18), 2019-2026.

10. Van Pelt, C. K.; Zhang, S.; Fung, E., Chu, I.; Liu, T.; Li, C.; Korfmacher, W. A.; Henion, J. A Fully Automated Nanoelectrospray Tandem Mass Spectrometric Method for Analysis of Caco-2 Samples. Rapid Commun. Mass Spectrom. 2003, 17(14), 1573-1578.

11. Zhang, S.; Van Pelt, C. K.; Wilson, D. B. Quantitative Determination of Noncovalent Binding Interactions Using Automated Nanoelectrospray Mass Spectrometry. Anal. Chem. 2003, 75(13), 3010-3018.

12. Ekstrom, S.; Malmstrom, J.; Wallman, L.; Lofgren, M.; Nilsson, J.; Laurell, T.; Marko-Varga, G. On-Chip Microextraction for Proteomic Sample Preparation of In-Gel Digests. Proteomics 2002, 2(4), 413-421.

13. Lamer, S.; Jungblut, P. R. Matrix-Assisted Laser DesorptionIonization Mass Spectrometry Peptide Mass Fingerprinting for Proteome Analysis: Identification Efficiency After On-Blot or In-Gel Digestion with and without Desalting Procedures. J. Chromatogr. B Biomed. Sci. Appl. 2001, 752(2), 311-322.

14. Lim, H.; Eng, J.; Yates, III; J. R., Tollaksen, S. L.; Giometti, C. S.; Holden, J. F.; Adams, M. W.; Reich, C. I.; Olsen, G. J.; Hays, L. G. Identification of 2D-Gel Proteins: A Comparison of MALDI/TOF Peptide Mass Mapping to $\mu$ LC-ESI Tandem Mass Spectrometry. J. Am. Soc. Mass Spectrom. 2003, 14(9), 957-970.

15. Pluskal, M. G.; Bogdanova, A.; Lopez, M.; Gutierrez, S.; Pitt, A. M. Multiwell In-Gel Protein Digestion and Microscale Sample Preparation for Protein Identification by Mass Spectrometry. Proteomics 2002, 2(2), 145-150.

16. Roepstorff, P.; Fohlman, J. Proposal for a Common Nomenclature for Sequence Ions in Mass Spectra of Peptides. Biomed. Mass Spectrom. 1984, 11(11), 601. 